


\title{
CO-OCURRENCIA DE MICROORGANISMOS Y SUS METABOLITOS TÓXICOS EN PRODUCTOS ALIMENTICIOS INFANTILES
}

\section{CO-OCURRENCE OF MICROORGANISMS AND TOXIC METABOLITES IN FOOD FOR CHILDREN}

\author{
Liliana Rojas C. ${ }^{1}$, Ángela Wilches F. ${ }^{2}$, Enrique Darghan C. ${ }^{3}$
}

\begin{abstract}
${ }^{1}$ Microbióloga. Especialista en Química Ambiental. Magister en Ciencia y Tecnología de Alimentos. U. de Pamplona, Facultad Ciencias Básicas, Departamento Microbiología, Grupo de Investigación en Microbiología y Biotecnología (GIMBIO). Pamplona. Norte de Santander. Colombia. olrojas@unipamplona.edu.co; ${ }^{2}$ Microbióloga. Especialista en Docencia Universitaria. Magister en Ciencias. Ph.D. en Bioética. Docente Titular, Facultad del Medio Ambiente y Recursos Naturales. Universidad Distrital Francisco José de Caldas. Bogotá. Colombia. amwilchesf@udistrital.edu.co; ${ }^{3}$ Ingeniero Agrónomo. Magíster en Estadística Aplicada. Ph.D. en Estadística. Universidad Nacional de Colombia. Bogotá. Colombia. aqedarghanc@unal.edu.co
\end{abstract}

Rev. U.D.C.A Act. \& Div. Cient. 18(1): 3-12, Enero-Junio, 2015

\section{RESUMEN}

La importancia de la alimentación en infantes hace cada vez más necesario el establecimiento de medidas de control que garanticen un consumo seguro de los alimentos. La calidad microbiológica de los alimentos infantiles comercializados en Pamplona (Colombia), revela que un $46,67 \%$ de los productos estudiados incumplen los parámetros de la Resolución del Ministerio de Salud 11488 de 1984. El 6,67\% de las muestras presentan mohos y levaduras, excediendo los valores permisibles. La presente investigación evaluó la coocurrencia de microorganismos coliformes totales, Escherichia coli, aerobios mesófilos, mohos y levaduras, junto a las micotoxinas: aflatoxinas, zearalenona y deoxinivalenol en muestras de alimentos pueriles. En la cuantificación de las micotoxinas, se aplicaron técnicas estandarizadas en el Laboratorio de Toxicología de la Universidad Nacional de Colombia. Con 30 muestras, se establecieron tres tipos de coocurrencia, atendiendo al efecto potencial nocivo de los contaminantes utilizando un análisis de clusters jerárquico. Los tipos de coocurrencia, se asociaron al origen de la contaminación: biológica $33,34 \%$, con solo microorganismos, química $3,33 \%$, con solo micotoxinas y biológico - química 3,33\%. El $40 \%$ no presentó contaminación relevante según normatividad colombiana. La permanencia de esta co-ocurrencia en otras investigaciones servirá de bioindicador para la detección de diversos agentes tóxicos con los que suele co-ocurrir, permitiendo su control en productos de consumo infantil, mediante la utilización de métodos preventivos inhibidores del crecimiento de hongos toxicogénicos o a través de la detoxificación de los alimentos.
Palabras clave: Aflatoxinas, alimentos infantiles, cromatografía, deoxinivalenol, microorganismos, zearalenona.

\section{SUMMARY}

The importance of nutrition in infants makes it increasingly necessary to establish control measures to ensure safe food consumption. The microbiological quality of baby foods marketed in Pamplona (Colombia), reveals that $46.67 \%$ of the products studied violate the parameters of the Resolution of the Ministry of Health $\mathrm{N}^{\circ} 11488$ of $1984.6 .67 \%$ of the samples presented molds and yeasts exceeding the permissible values. This research assessed the co-occurrence of total coliforms, Escherichia coli, aerobic mesophilic bacteria, molds and yeasts; mycotoxins: aflatoxin, zearalenone and deoxynivalenol in baby food samples. For quaintification of mycotoxins standardized techniques were applied in the Toxicology Laboratory of the National University of Colombia. 30 samples with three types of co-occurrence in response to potential harmful effects of pollutants using a hierarchical cluster analysis were established. The co-occurrence rates associated to the source of pollution were biological $33.34 \%$ with only microorganisms, chemical only $3.33 \%$ mycotoxins and biological - chemical 3.33\%. No significant contamination according to Colombian law was observed in a $40 \%$. The steadiness of this co-occurrence in other research can serve as a bio-indicator for the detection of various toxic agents, which often co-occur, allowing its control in products consumed by infants, employing preventive inhibitory growth methods of toxigenic fungi or food detoxification. 
Key words: Aflatoxins, infant food, chromatography, deoxynivalenol, microorganisms, zearalenone.

\section{INTRODUCCIÓN}

Cuando se evalúa la calidad de un alimento es necesario determinar la presencia de microorganismos y metabolitos secundarios, como micotoxinas, entre otros, los cuales, pueden ser causantes de enfermedades transmitidas por alimentos u ocasionar daños severos, tales como depresión del sistema inmunológico, alergias, daño renal y cáncer (CanoSancho et al. 2012). La exposición a las micotoxinas se produce, generalmente, por ingestión, pero también por contacto cutáneo o inhalación. Dichos efectos sobre la salud, se conocen como micotoxicosis, cuya gravedad depende de la toxicidad de la micotoxina, grado de exposición, edad y estado nutricional del individuo (Moss, 1996). Las micotoxinas son producidas por hongos que pueden contaminar los cereales y otros alimentos para consumo humano y animal. Los principales mohos filamentosos, productores de micotoxinas, pertenecen a los géneros Aspergillus, Fusarium y Penicillium (Nielsen et al. 2009). La contaminación de alimentos con micotoxinas ocurre en todo el mundo, dando lugar a graves riesgos en la salud humana y animal (Njobeh et al. 2009). Los factores que contribuyen a la presencia y producción de las micotoxinas en los alimentos y los piensos están asociados a las condiciones ambientales y ecológicas, aunado a las prácticas de almacenamiento (Zahin, 2011).

Se ha reportado que las micotoxinas generan pérdidas anuales millonarias a nivel global, involucrando deterioro de alimentos y provocando déficits económicos muy importantes, debido al rechazo de lotes contaminados, reducción de la productibilidad de los animales, aumento del costo por inspección, prevención y análisis, así como también por la compensación por reclamaciones y demandas (Zahin, 2011; Castillo \& Durán de Bazúa, 2004).

En la última década, internacionalmente ha surgido especial atención al estudio de la presencia de micotoxinas en alimentos para infantes, debido a que ellos son población vulnerable y a que su dieta esta mayormente restringida a alimentos conocidos, como "fórmulas infantiles" que utilizan como base al trigo, el maíz y la avena, cereales susceptibles a la contaminación con hongos, especialmente, de los géneros encontrados como productores de las siguientes toxinas: Aflatoxinas (AFLAS), Zearalenona (ZEA) y Deoxinivalenol (DON) (Li et al. 2014; Juan et al. 2014; Kimanya et al. 2014), siendo causantes de diversas enfermedades, lo que implica, a su vez, un problema de salud pública. Adicionalmente, otro aspecto que se viene estudiando es el de co-ocurrencia de las micotoxinas, lo que significa que diferentes micotoxinas ya sea de la misma o de diversas especies de hongos, pueden ser encontradas de manera simultánea en un producto (Li et al. 2014; Juan et al. 2014; Kimanya et al. 2014; Zafar et al. 2014); sin embargo, la implicación de esto, en la seguridad alimentaria, casi no se conoce, ya que es poca la información que existe sobre la interacción entre dos o más de estas moléculas y sus consecuencias ,a nivel humano. La premisa anterior justifica la necesidad de ampliar los estudios relacionados con la co-ocurrencia de las micotoxinas; por ello, en este estudio, se evaluó la co-ocurrencia de tres de ellas, a saber: AFLAS, ZEA y DON, junto con la presencia de coliformes totales, E. coli, aerobios mesófilos, mohos y levaduras, en alimentos de consumo infantil muestreados en la ciudad de Pamplona-Colombia. Los análisis referidos incluyeron los exámenes fundamentales para conocer las condiciones generales sobre la seguridad y calidad microbiológica de las muestras utilizadas, indicando que estos productos estuvieron expuestos a condiciones que pudieran haber introducido microorganismos patógenos y permitido la multiplicación de especies infecciosas y/o toxigénicas (Roberts et al. 2000). El principal objetivo de la utilización de microorganismos, como indicadores de prácticas no sanitarias, es revelar defectos de tratamiento, que lleven consigo un peligro potencial.

A continuación se presenta información relevante a las toxinas estudiadas:

Aflatoxinas (AFLAS). Son producidas principalmente por tres especies del hongo Aspergillus, a saber: A. flavus, A. parasiticus y A. nomius (Greppy, 2002) y se pueden encontrar en el suelo, en las plantas, en los restos de animales, en granos y en semillas de maíz y maní. Se han identificado veinte diferentes AFLAS, encontrando que B1, B2, G1 y G2 son las contaminantes en alimentos y piensos, originadas de manera natural (Decastelli et al. 2007). Otras AFLAS como M1, M2, P1, Q1, aflatoxicol, ocurren como productos metabólicos de sistemas microbianos o animales (Smith \& Ross, 1991). Las AFLAS son altamente tóxicas y actúan como sustancias mutagénicas, teratogénicas y carcinogénicas, por ello, la Agencia Internacional para la Investigación en Cáncer (IARC), las incluyó como compuestos carcinogénicos primarios (IARC, 2012). Entre las principales manifestaciones asociadas a la exposición de AFLAS, están el daño hepático y renal; mutagénesis, por la mutación en el codón 249 del gen TP53, en casos de carcinoma hepatocelular (Szymanska et al. 2004); la teratogénesis es atribuible al cruce de la placenta humana, lo que puede crear malformaciones fetales, inmunosupresión, alteración de la funcionalidad del sistema del complemento y enmudece la acción de la IgA y la IgG, además de genotoxicidad, por la formación de aductos con el ADN y con la albúmina que induce a la mutación de genes y alteraciones cromosómicas, como intercambio entre cromátidas hermanas y recombinación (Eley,1992). Otros efectos presuntamente relacionados involucran el daño al hígado, Síndrome de Reye-Kwashiorkor, cirrosis en niños, 
gastritis crónica, y daños respiratorios (Castillo \& Durán de Bazúa, 2006).

Deoxinivalenol (DON). Uno de los más comunes, pero a la vez menos tóxicos de los tricotecenos (Cano-Sancho et al. 2012) es producido por hongos de los géneros Fusarium, Myrothecium, Cephalosporium, Stachybotrys y Trichoderma (Beremand \& McCormick, 1992). Los más importantes en los alimentos son los producidos por Fusarium y, entre ellos, los relacionados con micotoxicosis de que afectan humanos y animales; se encuentra en granos de trigo, de cebada, de maíz, de avena, de centeno y, en menor proporción, en arroz y sorgo. La contaminación con estos metabolitos está restringida a los cereales y suele ocurrir en las etapas de pre-cosecha y no es usual en almacenaje (Logrieco et al. 2003).

El principal síndrome provocado por DON es gastroentérico y afecta el aparato digestivo. Esta toxina, también tiene una potente actividad inmunosupresiva y reduce el crecimiento en niños. La toxina actúa sobre el sistema nervioso central causando un síndrome emético y rechazo del alimento (Moss, 1996; Speijers \& Speijers, 2004). El DON fue clasificado en 1993 como agente carcinogénico del grupo 3 por la IARC (Li et al. 2014).

Zearalenona (ZEA). Es producida por especies del hongo Fusarium, comúnmente F. graminearum (Jurado, 1989). Otras especies productoras de la toxina son F. moniliforme, F. culmorum, F. sporotrichioides, F. oxysporum y F. semitectum (Kos et al. 2013). Aparece en maíz, en granos de trigo, en avena, en cebada, en sorgo, en mijo así, como heno, ensilados y alimentos balanceados (Díaz, 1996). La ZEA estimula receptores estrogénicos, por lo tanto, la toxicidad está asociada con problemas reproductivos en cerdos y ovejas, observándose problemas de fertilidad, lo que posiblemente, también se pueda dar en humanos (Gromadzka et al. 2009). Puede ser un importante agente etiológico de intoxicación en niños y fetos expuestos a la micotoxina, con consecuencias, como telarquia y pubarquia prematura (CAST, 2003). La evaluación llevada a cabo por la IARC sobre el potencial carcinogénico de la ZEA, concluyó que no es clasificable por su carcinogenicidad (grupo 3) (IARC, 2013); sin embargo, estudios recientes han mostrado que la ZEA puede estimular, potencialmente, el crecimiento de células cancerosas, con receptores estrogénicos en glándulas mamarias de humanos; también se asocia como un posible factor involucrado en cáncer de cerviz (Blunden et al. 1991) y en algunos casos, de hiperestrogenismo humano (Milicevic et al. 2008).

\section{MATERIALES Y MÉTODOS}

Se analizaron un total de 30 muestras comerciales, 22 de procedencia nacional y ocho venezolanas, dado que se trata de zona de frontera. Su selección, se basó en los resultados obtenidos del muestreo por encuestas, aplicadas en establecimientos comerciales, destinados a la venta de productos alimenticios de consumo infantil; de igual forma, a padres de familia de niños lactantes (menores de 1 año) y niños de corta edad (mayores de 1 año y menores de 3 años). La encuesta evaluó el grado de preferencia de los productos de mayor consumo infantil comercializados en la ciudad, y permitió la clasificación de los alimentos, en cuatro categorías, elaborados a base de harinas: 1) crudas, 2) pre-cocidas, 3) instantáneas y 4) avenas. Un 38\% de los padres declararon su preferencia a estos complementos alimenticios, por su calidad, un $33 \%$, por su valor nutricional y, un $29 \%$, por los costos.

Análisis microbiológicos: Para coliformes totales y Eschericihia coli se utilizó método en tubo (LMX - Fluorocult), recomendado por la FDA (1995). Para el recuento de aerobios mesófilos, mohos y levaduras, se aplicó método en placa, recomendados por el Instituto Nacional de Salud y por el ICMSF (2000).

Análisis de micotoxinas: Las técnicas utilizadas fueron estandarizadas en el Laboratorio de Toxicología (Facultad de Medicina Veterinaria), de la Universidad Nacional de Colombia, basadas en técnicas reconocidas por la Asociación de Químicos Analíticos Oficiales AOAC, para análisis de alimentos y en el método descrito por (Trucksess et al. 1994), para AFLAS. La separación, la detección y la cuantificación de la ZEA, se efectuó por HPLC, según el método reportado por Bennet et al. (1985), el cual ha sido adoptado como un método oficial de análisis de la AOAC. Para DON, se realizó la extracción con una mezcla de acetonitrilo:agua, se purificó con columna de limpieza; el análisis cromatográfico; se realizó en columna de fase reversa con detección espectrofotométrica (detector ultravioleta a 220nm). Los resultados de las micotoxinas estudiadas se expresaron en $\mu \mathrm{g} / \mathrm{kg}$.

Los métodos estadísticos utilizados involucraron el componente descriptivo univariante y multivariante, por la naturaleza observacional del estudio, del cual, se destacó la medición de la prevalencia de las micotoxinas; además, se construyó una tabla de frecuencias, para identificar las muestras dentro de un conglomerado (cluster), con co-ocurrencia biológica, química, químico-biológica o sin contaminación. El análisis descriptivo multivariante generó un par de dendrogramas: uno por variables para asociar o discriminar las micotoxinas o microorganismos, según el método del vecino más lejano, usando la métrica de distancia Euclideana Cuadrada, con medidas estandarizadas, para corregir las unidades de medición. Se solicitaron tres conglomerados en la agrupación por variables, pues así se logró separar, según lo observado, los tres grupos principales de agentes patógenos. El segundo dendrograma, se realizó por observaciones para discriminar 
por muestras las que poseen cierto tipo de contaminación; se generaron cuatro conglomerados y, con estos, se pudo segregar la fuente de contaminación predominante y, por ende, el tipo de co-ocurrencia encontrada. Finalmente, se construyó una tabla de estadísticas descriptivas de tendencia central (promedio), dispersión (desviación típica) y dispersión relativa (coeficiente de variación), para las variables ambientales humedad (\%), $\mathrm{pH}$ y temperatura $\left({ }^{\circ} \mathrm{C}\right)$ según el tipo de co-ocurrencia.

\section{RESULTADOS Y DISCUSIÓN}

La figura 1 revela los resultados de los análisis microbiológicos: coliformes totales, E. coli, aerobios mesófilos y mohos y levaduras, realizados a las muestras de productos alimenticios. Los niveles de coliformes totales variaron entre 0 y 3,04Log(bacterias/g), siendo la media de 1,10Log(bacterias/g); en relación con $E$. coli, los niveles variaron entre 0 y $1,63 \log ($ bacterias $/ g$ ) la media fue de 0,17Log(bacterias/g); atendiendo al recuento total de microorganismos aerobios mesófilos, los niveles oscilaron entre 0 y 4,56Log(UFC/g) y los niveles de mohos y levaduras fluctuaron entre 0 y $3,64 \log (\mathrm{UFC} / \mathrm{g})$. El $56,67 \%$ de las muestras (17/30) incumplieron con los parámetros microbiológicos, estipulados por la Resolución del Ministerio de Salud No. 11488 de 1984, siendo los parámetros coliformes totales (11/30), 37\% y E. coli (4/30) 13,33\%, los que presentaron mayor porcentaje de incumplimiento, presentándose una alta probabilidad de padecimiento de enfermedades y un alto riesgo para la salud pública, hecho que sugiere mayor control por las entidades de salud.

En la figura 2 se presentan los resultados de la cuantificación de AFLAS (B1 y totales), DON y ZEA. Se detectaron AFLAS en el $10 \%$ de las muestras, con niveles entre $18,42 \mu \mathrm{g} / \mathrm{kg}$ y $71,25 \mu \mathrm{g} / \mathrm{kg}$ de AFB1. Los niveles de AFLAS superaron el valor máximo admisible por la legislación colombiana $(4,0 \mu \mathrm{g} /$ kg) y por la Unión Europea -UE- $(0,10 \mu \mathrm{g} / \mathrm{kg})$, para alimentos infantiles y alimentos elaborados a base de cereales, situación preocupante debido a su condición cancerígena, dado que estudios, a nivel mundial han revelado que por años se acumulan AFLAS en el ADN de las personas que consumen alimentos, en los cuales están presentes cereales (maíz, arroz, cebada, entre otros) y en aquellas personas susceptibles, que pueden desarrollar diferentes tipos de cáncer (hígado, colorrectal, de páncreas y de pulmón).

Estudios de monitoreo realizados en Colombia han revelado una prevalencia, relativamente, alta de AFB1. La prevalencia de AFLAS en este estudio, $10 \%$, coincide con un estudio de monitoreo de AFLAS, en alimentos de consumo humano realizado en Colombia, que mostró una prevalencia de AFB1 cercana al $10 \%$, aproximadamente, una de cada 10 muestras resultó positiva. Adicionalmente, a esta preocupante prevalencia, varias de las muestras positivas se asociaron a niveles superiores a $2 \mu \mathrm{g} / \mathrm{kg}$, nivel máximo de AFB1 aceptado en la mayoría de países (Díaz, 2005).

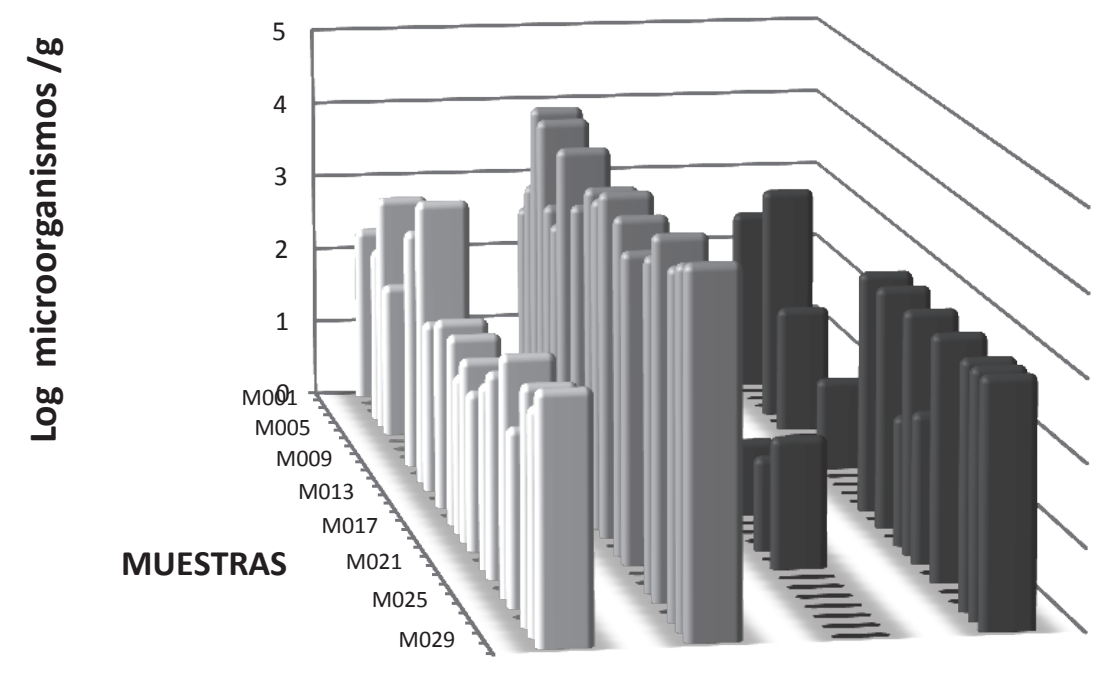

๑COLIFORMES TOTALES $\square$ E. coli $\square$ AEROBIOS MESÓFILOS $\sqcup$ MOHOS Y LEVADURAS

Figura 1. Co-ocurrencia de Microorganismos (Coliformes Totales, E. coli, Aerobios Mesófilos, Mohos y Levaduras). 


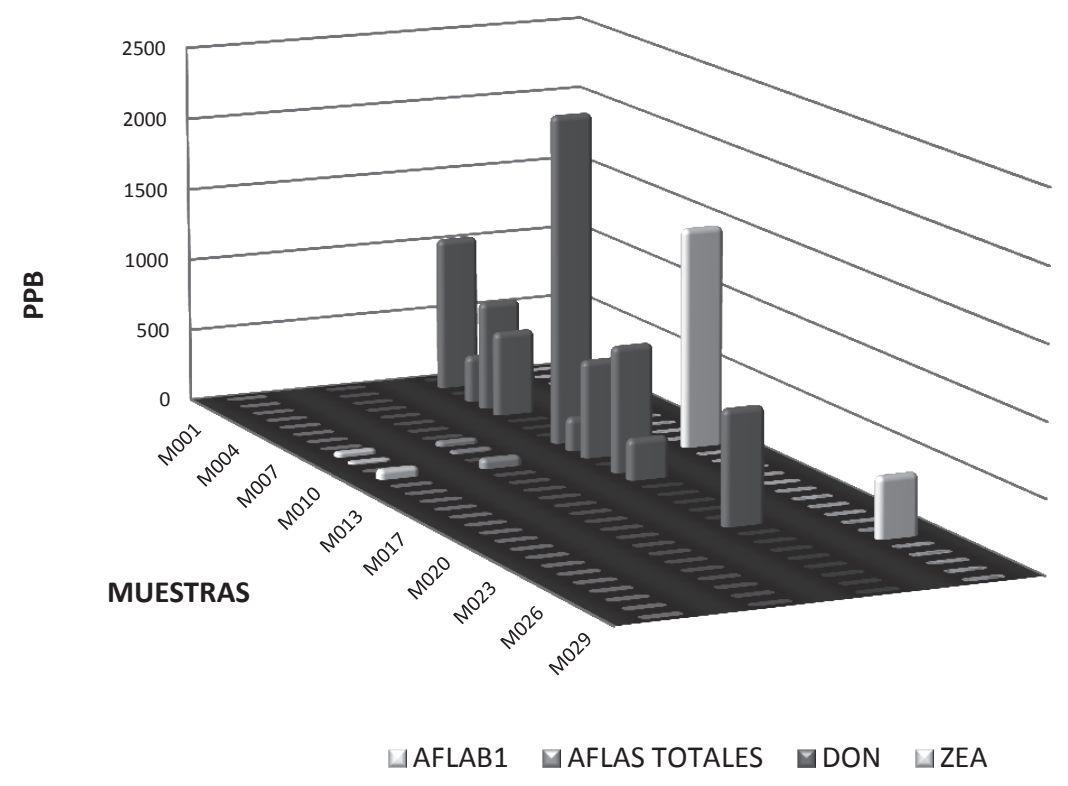

Figura 2. Co-ocurrencia de aflatoxinas, deoxinivalenol y zearalenona expresadas en partes por billón (PPB).

La prevalencia del DON fue de $36,67 \%$, en concentraciones entre $231,10 \mu \mathrm{g} / \mathrm{kg}$ y $2273,04 \mu \mathrm{g} / \mathrm{kg}$; todas las muestras superaron el valor máximo legal en Colombia y por la UE $(200 \mu \mathrm{g} / \mathrm{kg})$, lo cual, es preocupante, ya que el riesgo para la poblacional infantil, por la exposición a esta micotoxina puede, interferir negativamente en el crecimiento normal. Esta prevalencia es alta, comparada con los análisis de tricoticenos tipo A y B realizados rutinariamente en el Laboratorio de Toxicología de la Universidad Nacional, indicando que la prevalencia de estos compuestos puede ser cercana al 10\% (Díaz, 2005). En el contexto global, estudios realizados en Europa, muestran la proliferación de micotoxinas, tanto en los granos de trigo, como en productos procesados de panadería, encontrando concentraciones de DON en harina de trigo de $333 \mu \mathrm{g} / \mathrm{kg}$ y valores inferiores en pan, de 275 a $314 \mu \mathrm{g} / \mathrm{kg}$ (Bergamini et al. 2010; Duarte et al. 2010; Pascale et al. 2011).

La ZEA tuvo una prevalencia de $6,67 \%$, en concentraciones entre 421,34 y $1518,22 \mu \mathrm{g} / \mathrm{kg}$, superando los valores legales Colombianos y de la UE $(20 \mu \mathrm{g} / \mathrm{kg})$. Este valor fue relativamente alto, comparado con un estudio realizado en 1999 (Díaz, 2005), donde se halló una prevalencia global del 2,3\% (7/300), de ZEA, en muestras de alimentos de consumo humano (maíz y derivados, arroz, trigo, avena, cebada). La prevalencia en maíz procesado fue del $6,7 \%(4 / 60)$ y los niveles de ZEA, en las muestras positivas, oscilaron entre 35 y $134 \mu \mathrm{g} / \mathrm{kg}$.
La figura 3 muestra el resultado del análisis de conglomerados por variables de forma global, específicamente, las que están asociadas a las micotoxinas y los microorganismos. El primer conglomerado reúne a las micotoxinas AFLAS, DON y ZEA y, el segundo a los microorganismos: aerobios mesófilos, coliformes totales y mohos-levaduras. La E. coli, se separa en un conglomerado con un sólo miembro, con una prevalencia del 13,33\%; los otros microorganismos presentaron prevalencias superiores: $40 \%$, en el caso de coliformes totales; $60 \%$, para aerobios mesófilos y, $63,33 \%$ para mohos y levaduras. La aglomeración anterior justifica el estudio de la co-ocurrencia, pues es precisamente en los conglomerados de micotoxinas y de microorganismos donde se conforma el agrupamiento.

En la figura 4, se presenta el dendograma por observaciones, donde se discriminan las observaciones en cuatro conglomerados, que pudieron ser etiquetados, según el efecto potencial que pudieron tener en una población pueril. La muestra 12, perteneciente al conglomerado 4, se aísla completamente de las otras muestras y es precisamente en esta donde ocurre, de manera simultánea, la presencia de AFLAS, ZEA y DON como micotoxinas, además de aerobios mesófilos, coliformes totales y mohos y levaduras, como microorganismos; este agrupamiento le da una naturaleza diferente, por lo que puede aseverarse que es la única muestra con una contaminación de tipo biológico y químico (bioquímica). La muestra 10, también se separa de forma aislada 


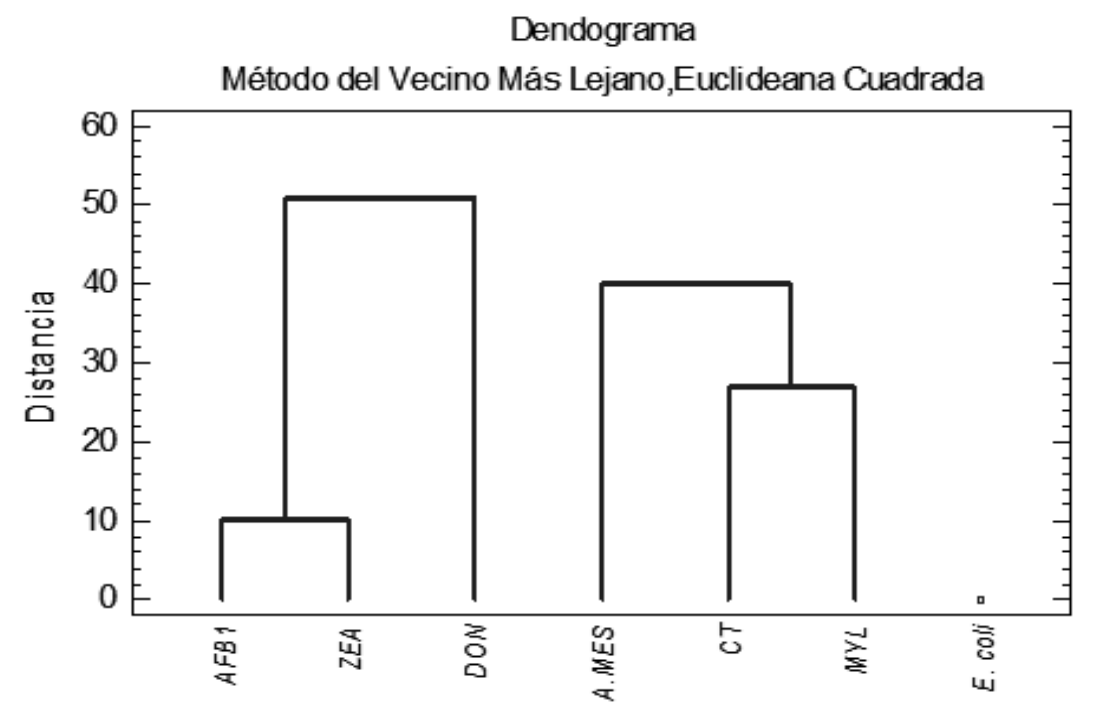

Figura 3. Dendrograma por micotoxinas y microorganismos.

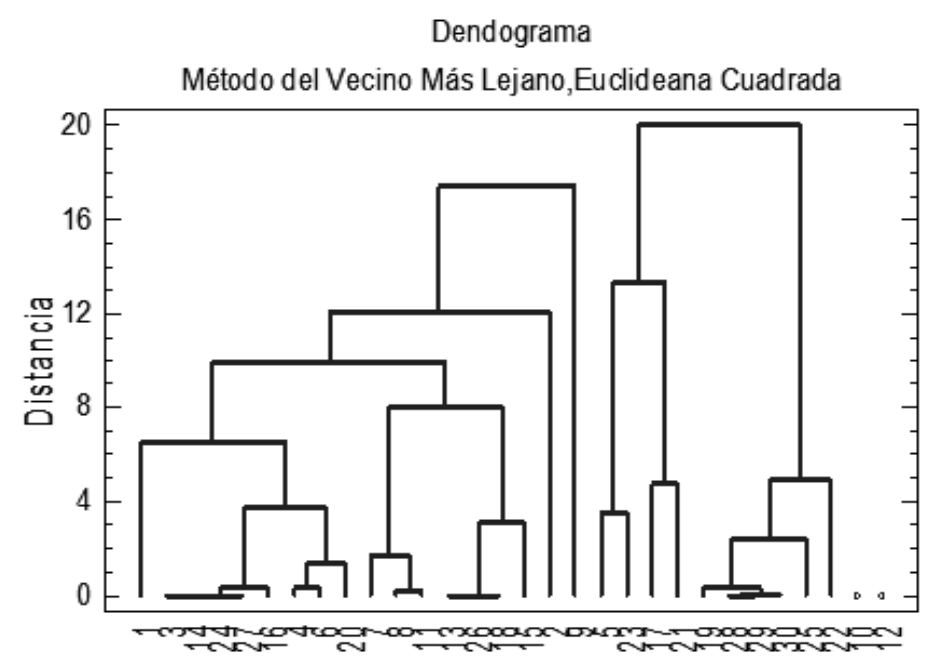

Figura 4. Dendrograma por micotoxinas y microorganismos, según las muestras.

en el conglomerado 3 y esta muestra es la única que posee una contaminación de tipo químico parcial (pues se excluye ZEA) y no biológico, pues aunque se midieron valores de aerobios mesófilos, coliformes totales y mohos y levaduras, sus valores cayeron en rangos, aceptados por la Resolución Ministerial $N^{\circ} 11488$.
El conglomerado 2 posee el 33,33\% de las muestras y lo conforman muestras con contaminación, solamente del tipo biológico. Finalmente, el conglomerado con la mayor cantidad de muestras lo conforman el $60 \%$ de ellas, presentándose muestras con recuentos aceptables de mohos y levaduras y aerobios mesófilos y, de igual forma, se percibe la presencia de DON, en valores dentro de rangos aceptables. 
La tabla 1 resume la descripción de los conglomerados, su distribución porcentual y las muestras que lo conforman, así como la etiqueta asignada al conglomerado, según el tipo de contaminación existente o predominante, destacándose los tres últimos conglomerados, como los que exhiben el patrón de co-ocurrencia, biológica, química y biológico-química.
La tabla cruzada multidimensional (Tabla 2) muestra las características descriptivas de los factores ecofisiológicos evaluados, según la categorización del tipo de co-ocurrencia; donde aparece un guión, se debe a la presencia de una sola muestra, por lo que no es medible ni la desviación estándar ni el coeficiente de variación.

Tabla 1. Distribución de las muestras por conglomerado y categorización, según el efecto potencial nocivo en la población infantil.

\begin{tabular}{|c|c|c|c|}
\hline Conglomerado & Porcentaje de Muestras & Muestra(s) & Etiqueta \\
\hline 1 & 60,00 & $1-4,6-9,11,13-16,18,20,22$ & No contaminado \\
\hline 2 & 33,34 & $5,17,19,21-23,25,28-30$ & $\begin{array}{c}\text { Contaminación } \\
\text { Biológica }\end{array}$ \\
\hline 3 & 3,33 & 10 & $\begin{array}{c}\text { Contaminación } \\
\text { Química }\end{array}$ \\
\hline 4 & 3,33 & 12 & $\begin{array}{c}\text { Contaminación } \\
\text { biológica-química. }\end{array}$ \\
\hline
\end{tabular}

Tabla 2. Características descriptivas de las variables ambientales de las muestras de productos alimenticios infantiles.

\begin{tabular}{|c|c|c|c|c|}
\hline $\begin{array}{c}\text { Variable } \\
\text { Ecofisiológica }\end{array}$ & \multicolumn{2}{|c|}{$\begin{array}{c}\text { Humedad } \\
\%\end{array}$} & \multirow{2}{*}{$\begin{array}{c}\mathrm{pH} \\
5,95\end{array}$} & \multirow{2}{*}{$\begin{array}{c}{ }^{\text {To }} \mathrm{T} \\
18,67\end{array}$} \\
\hline \multirow{3}{*}{ Químico-biológica } & Promedio & 6,13 & & \\
\hline & Desviación estándar & - & - & - \\
\hline & $\begin{array}{l}\text { Coeficiente de } \\
\text { variación }\end{array}$ & - & - & - \\
\hline \multirow{3}{*}{ Química } & Promedio & 7,72 & 5,96 & 19,40 \\
\hline & Desviación estándar & - & - & - \\
\hline & $\begin{array}{l}\text { Coeficiente de } \\
\text { variación }\end{array}$ & - & - & - \\
\hline \multirow{3}{*}{ Biológica } & Promedio & 10,02 & 5,70 & 19,40 \\
\hline & Desviación estándar & 3,17 & 0,76 & 0,51 \\
\hline & $\begin{array}{l}\text { Coeficiente de } \\
\text { variación }\end{array}$ & $31,65 \%$ & $13,35 \%$ & $2,61 \%$ \\
\hline \multirow{3}{*}{ Ninguna } & Promedio & 6,68 & 5,97 & 18,84 \\
\hline & Desviación estándar & 2,99 & 0,64 & 0,51 \\
\hline & $\begin{array}{l}\text { Coeficiente de } \\
\text { variación }\end{array}$ & $44,83 \%$ & $10,76 \%$ & $2,73 \%$ \\
\hline
\end{tabular}


En cuanto a las variables ecofisiológicas, el valor promedio del contenido de agua, 7,82\%, está dentro de lo estipulado por la legislación colombiana; el $10 \%$ de las muestras superan el valor máximo permitido. El valor medio del $\mathrm{pH}$ fue de 5,88 , mientras que la temperatura presentó un valor medio de $19^{\circ} \mathrm{C}$, favoreciendo el crecimiento fúngico. Los valores de $\mathrm{pH}$ y temperatura son similares en los cuatro tipos de coocurrencia; sin embargo, la humedad resultó más elevada en las muestras de co-ocurrencia biológica. El coeficiente de variación para la co-ocurrencia biológica es el más elevado, junto a la categoría de ausencia de contaminación, pero sólo en la variable humedad; no obstante, para los tipos de ocurrencia, no es una medida que pudiera perturbar los resultados obtenidos. La co-ocurrencia de estas micotoxinas es probable que se presente, porque la mayoría de los hongos son capaces de producir simultáneamente varias micotoxinas o porque los productos analizados presentan condiciones ecofisiológicas, que permiten la proliferación de varios hongos. La co-exposición prolongada a las AFLAS, DON, y ZEA podría aumentar el riesgo de varias enfermedades crónicas.

La co-ocurrencia de micotoxinas en productos alimenticios para infantes viene siendo estudiada por diversos autores, en especial, en la zona del Mediterráneo, probablemente, debido a que en esta región geográfica se dan las condiciones climatológicas favorables para el desarrollo de hongos productores de micotoxinas; sin embargo, se concluye que aún es necesario el desarrollo de nuevas investigaciones sobre el tema y sobre todo se requiere mayor regulación por parte de los entes de control en cada país, para minimizar sus afectaciones en la población infantil (Juan et al. 2014).

Agradecimientos: A la Universidad de Pamplona, por la financiación del proyecto. A la Universidad Nacional de Colombia, en su nombre, al Laboratorio de Toxicología (Facultad de Medicina Veterinaria), por la aplicación de los métodos analíticos para cuantificar las micotoxinas objeto de estudio. Financiación: Este estudio fue financiado por la Universidad de Pamplona, Pamplona, Norte de Santander, Colombia. Conflicto de intereses: El manuscrito fue preparado y revisado con la participación de todos los autores, quienes declaramos que no existe conflicto de intereses que ponga en riesgo la validez de los resultados presentados.

\section{BIBLIOGRAFÍA}

1. BENNETT, G.A.; SHOTWELL, O.L.; KWOLEK, W.F. 1985. Liquid Chromatographic Determination of a-zearalenol and zearalenone in com: Collaborative Study. J. Assoc. Anal. Chem. 68:958-961.

2. BEREMAND, M.N.; McCORMICK, S.P. 1992. Biosynthesis and regulation of trichothecene production by
Fusarium species. In: Bhatnagar, D.; Lillehoj, E.B.; Arora, D.K. (eds). Handbook of applied mycology. Vol 5. 8a. Ed. Marcel Dekker., Inc, New York. p.359383.

3. BERGAMINI, E.; CATELLANI, D.; DALLASTA, C.; GALAVERNA, G.; DOSSENA, A.; MARCHELLI, R. 2010. Fate of Fusarium mycotoxins in the cereal product supply chain: the deoxynivalenol (DON) case within industrial bread-making technology. Food Additives \& Contaminants: Part A. 27(5):677-687.

4. BLUNDEN, G.; ROCH, O.G.; ROSERS, D.J.; COKER, R.D.; BRADBURN, N.; JHON, A.E. 1991. Mycotoxins in Food. Med. Lab. Sci. 48:271-282.

5. CANO-SANCHO, G.; RAMOS, A.J.; MARIN, S.; SANCHIS, V. 2012. Presence and co-ocurrence of aflatoxins, deoxynivalenol, fumonisins and zearalenone in gluten-free and ethnic foods. Food Control. 26(2):282-286.

6. CAST COUNCIL FOR AGRICULTURAL SCIENCE AND TECHNOLOGY 2003. Mycotoxins: Risks in plant, animal, and human system; Task Force Report Ames, IA. 139:48-58.

7. CASTILLO, U.P.; DURÁN DE BAZÚA, M.C. 2004. Aflatoxinas en maíz amarillo usado para elaborar jarabes de fructosa: ¿Existen riesgos para la salud? Industria Aliment. 26(4):28-35.

8. CASTILLO, U.P.; DURÁN DE BAZÚA, M.C. 2006. Las micotoxinas: metabolitos secundarios de los hongos filamentosos. Ciencia, Tecnología y Sociedad. Ed. Quím. 17(2):122-128.

9. DECASTELLI, L.; LAI, J.; GRAMAGLIA, M.; MONACO, A.; NACHTMANN, C.; OLDANO, F. 2007. Aflatoxins ocurrence in milk and feed in Northern Italy during 2004-2005. Food Control. 18(10):1263-1266.

10. DÍAZ, G.J. 1996. Micotoxinas y micotoxicosis en salud humana y animal. Vet. al Día. Primera parte. 2(1):2834.

11. DÍAZ, G.J. 2005. VII Congreso Latinoamericano de Microbiología e Higiene de Alimentos COLMIC. Bogotá. Mayo 18-21, 2005, Bogotá, Colombia.

Micotoxinas y micotoxicosis de importancia en salud humana en Colombia. U.N. de Colombia. 13p.

12. DUARTE, S.; LINO, C.; PEÑA, A. 2010. Mycotoxin food and feed regulation and especific case of ochratoxin 
A: a review of the worldwide status. Food Add. Contamin. 27(10):1440-1450.

13. ELEY, R. 1992. Intoxicaciones Alimentarias de Etiología Microbiana. Editorial Acribia. Zaragoza. España. 232p.

14. FDA. Food and Drug Administration U.S. 1995. Bacteriological Analytical Manual for Food. $8^{\text {th }}$ edition. AOAC International, Gaithenrsburg, M.D.

15. GREPPY, E.E. 2002. Update of survey, regulation and toxic effect of mycotoxins in Europe. Toxicol. Letters. 127(1-3):19-28.

16. GROMADZKA, K.; WASKIEWICZ, A., GOLINSKI, P.; SWIETLIK, J. 2009. Ocurrence of estrogenic mycotoxin - zearalenone in aqueous environmental samples with various NOM content. Water Res. 43:10511059.

17. IARC, International Agency for research on Cancer. 2012. IARC monographs on the evaluation of carcinogenic risks to humans. p.1-104.

18. IARC, International Agency for research on Cancer. 2013. IARC releases the latest global cancer trends in five continents. Press Release $\mathrm{N}^{\circ} 222$.

19. ICMSF, International Commission on Microbiological Specification for Food. 2000. Microorganismos de los alimentos. Técnica de análisis microbiológico. Vol 1. Editorial Acribia. Zaragoza, España. p.5-13.

20. JUAN, C., RAIOLA, A., MAÑES, J.; RITIENI, A. 2014. Presence of micotoxins in comercial infant formulas and baby foods from Italian market. Food Control. 39:227-236.

21. JURADO, R. 1989. Toxicología Veterinaria. Segunda Edición. Salvat. Madrid, España. 618p.

22. KIMANYA, M.; SHIRIMA, C.; MAGOHA, H.; SHEWIYO, D.; De MEULENAER, B.; KOLSTEREN, P.; GONG, Y. 2014. Co-exposures of aflatoxins with deoxynivalenol and fumonisins from maize based complementary foods in Rombo, Northern Tanzania. Food Control. 41:76-81.

23. KOS, J.; JASNA, M.; ELIZABETH, J.H.; BOJANA, S. 2013. Natural ocurrence of aflatoxins in maize harvested in Serbia during 2009-2012. Food Control. 34(2013):31-34.
24. LI, R.; XU, W.; TING, Z.; DONGXU, Y.; QI, W., YU, Z. 2014. Ocurrence of four mycotoxins in cereal and oil products in Yangtze Delta region of China and their food safety risks. Food Control. 35(1):117-122.

25. LOGRIECO, A.; BOTTALICO, A.; MULÉ, G.; MORETTI, A.; PERRONE, G. 2003. Epidemiology of toxigenic fungi and their associated mycotoxins for some Mediterranean crops. Eur. J. Plant Path. 109:645-667.

26. MILICEVIC, D.; JURIC, V.; STEFANOVIC, S.; JOVANOVIC, S. 2008. Survey of slaughterd pigs for occurrence of ochratoxin A and porcine nephropathy in Serbia. Int. J. Mol.Sci. 9:2169-2183.

27. MOSS, M.O. 1996. Centenary review: mycotoxins. Mycol. Res. 100(5):513-523.

28. NIELSEN, K.F.; MOGENSEN, J.M.; JOHANSEN, M.; LARSEN, T.O.; FRISVAD, J.C. 2009. Review of secondary metabolites and mycotoxins from the Aspergillus niger group. Analyt. Bioanalyt. Chem. 395(5): 1225-1242.

29. NJOBEH, P.B.; DUTTON, M.F.; KOCH, S.H.; CHUTURGOON, A.; STOEV, S.; SEIFERT, K. 2009. Contamination with storage fungi of human food from Cameroon. Int. J. Food Microbiol. 135(3):193-198.

30. PASCALE, M.; HAIDUKOWSKI, M.; LATTANZIO, V.M.; SILVESTRI, M.; RANIERI, R., VISCONTI, A. 2011. Distribution of T-2 and HT-2 toxins in milling fractions of durum wheat. J. Food Prot. 74(10):17001707.

31. ROBERTS, D.; HOOPER, W.; GREENWOOD, M. 2000. Microbiología de los Alimentos. Editorial Acribia, S.A. Zaragoza, España. 276p.

32. SMITH, J.E.; ROSS, K. 1991. The toxigenic Aspergilli. En: Smith, J.; Henderson, R. (Eds) Mycotoxins and Animal Foods. CRC Press, Boca Ratón, Florida. p.101-108.

33. SPEIJERS, G.J.; SPEIJERS, M.H. 2004. Combined toxic effects of mycotoxins. Toxicol. Letters. 153(1):91-98.

34. SZYMANSKA, K.; LESI, O.A., KIRK, G.D.; SAM, O.; TANIERE, P.; SCOAZEC, J.Y. 2004. Ser-249TP53 mutation in tumour and plasma DNA of hepatocellular carcinoma patients from a high incidence area in the Gambia, West Africa. Int. J. Cancer. 110(3):374-379. 
35. TRUCKSESS, M.W.; STACK, M.E.; NESHEIM, S. 1994. Multifunctional column coupled with liquid chromatography for determination of aflatoxins B1, B2, G1 and G2 in com, almonds, Brazil nuts, peanuts, and pistachio nuts: Collaborative Study. J. AOAC Int. 77(6):1512-1521.

36. ZAFAR, S., RAFIQUE, M.; JINAP, S.; RASHID, U. 2014. Detection of aflatoxins and zearalenone contamination in wheat derived products. Food Control. 35(1):223-226.
37. ZAHIN, M. 2011. Impact of mycotoxins on humans and animals. J. Saudi Chem. Soc. 15(2):129-144.

Recibido: Abril 1 de 2014

Aceptado: Enero 19 de 2015

Cómo citar:

Rojas C., L.; Wilches F., Á.; Darghan C., E. 2015. Co-ocurrencia de microorganismos y sus metabolitos tóxicos en productos alimenticios infantiles. Rev. U.D.C.A Act. \& Div. Cient. 18(1): 3-12. 